\title{
Evaporation and Explosion of Liquid Drops on a Heated Surface
}

\author{
N. Zhang* and W.-J. Yang \\ Department of Mechanical Engineering \& Applied Mechanics, University of Michigan. Ann Arbor, Mich. 48 109. USA
}

\begin{abstract}
The literature pertinent to various aspects of drop evaporation on a heated surface is reviewed. Both the laser shadowgraphic and direct photographic methods are employed to study thermal stability and flow structures in evaporating drops in all heating regimes. It is revealed that four flow regions exist in stable and unstable type drops at low liquid-film type vaporization regime. As the surface temperature is raised, the flow regions reduce to two. In the nucleate-boiling type vaporization regime, the interfacial flow structure changes due to a reduction in the Marangoni number as well as the dielectric constant of the liquid. An evidence of bubble growth in the drops is disclosed. The micro explosion of drops is found to occur in the transitionboiling type heating range. No drop explosion takes place in the spheriodal vaporization regime except when the drop rolls on to a microscratch on the heating surface. It is concluded that the mechanisms for triggering drop explosion include the spontaneous nucleation and growth phenomena and the destabilization of film boiling.
\end{abstract}

\section{Introduction}

Liquid drops are commonly seen both in nature and in human activities, particularly in many industrial and biological systems and processes. The diminution of liquid droplets by evaporation is of great importance in various areas of technology, for example, the internal combustion engine, aerosols spray combustion, spray drying, directcontact heat exchange, spray cooling of metals, spray cooling of nuclear reactor cores during emergency shut down, and others. Interest in these areas has prompted a considerable number of investigations into various aspects of small drop phenomena.

In compression ignition engines, gas turbines and other oil-fired appliances, there is literally no way of preventing the fuel drops from hitting the walls of the combustion chamber. In a number of technological processes such as quenching, cooling of a cutting metal, spray cooling of metals, and spray cooling of nuclear reactor cores during emergency shut down, etc., liquid flows freely over a

* Visiting Scholar on leave from the Department of Thermal Engineering, Tsinghua University, Beijing, China heated surface in the form of small drops or large portions of liquid.

Figure 1 shows a typical curve illustrating the dependence of the total vaporization time of a liquid drop on the temperature of the heating surface $T_{w}$ (Tamura and Tanasawa, 1959). Its corresponding pool boiling curve is included in Fig. 1. When $T_{w}$ is below the boiling temperature of the liquid $T_{b}$, liquid drops spread into a thin layer and evaporate slowly. This range $a-b$ is called the liquidfilm type vaporization regime in which the heat is transferred from the surface to the liquid by convection. At the point $\mathrm{b}$ when $T_{w}$ is equal to $T_{b}$, nucleate boiling is observed in the spreading liquid with a vapor bubble arising in the central part of the drop. With a further increase in $T_{w}$, the number of nucleation sites increases and
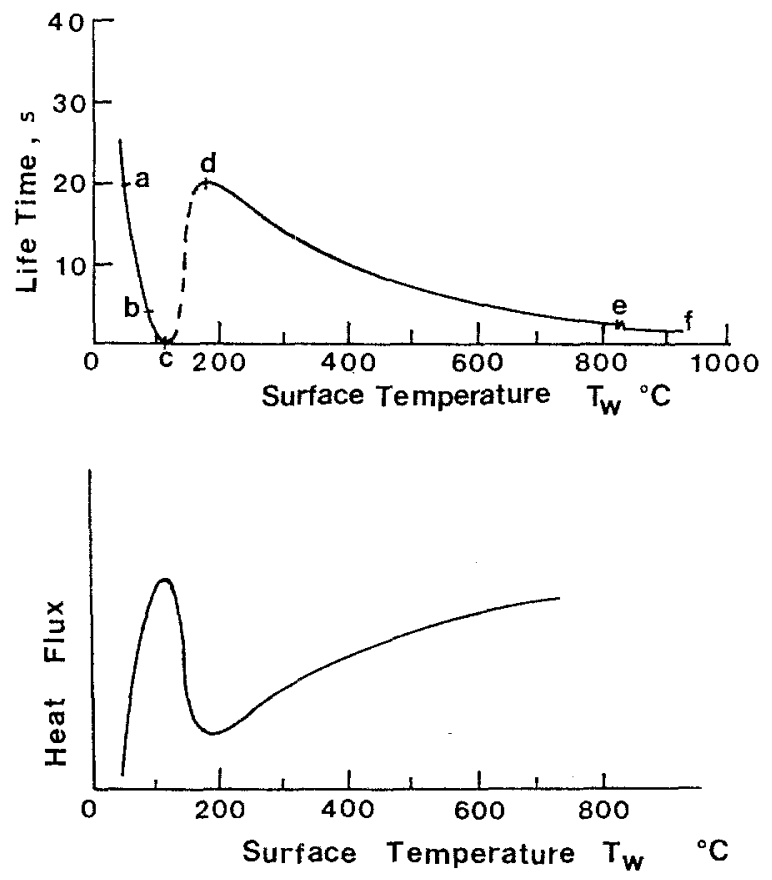

Fig. 1. A typical curve for the evaporation of benzene drops with the initial diameter of $2.14 \mathrm{~mm}$ and its corresponding pool-boiling curve 
the total droplet lifetime decreases accordingly. When the maximum boiling rate is reached at point $c$, called the maximum boiling rate point, the liquid forms a spherical drop which intermittently makes contact with the surface and vaporizes under turbulent conditions. The range $b-c$ is called the nucleate-boiling type vaporization regime. On subsequent increase of $T_{w}$, the frequency of contact between the drop and the surface decreases and the time required for complete vaporization increases. This indicates a decrease in the rate of heat transfer from the heating surface to the drop which is caused by the formation of a vapor film between the drop and the heating surface. The drop lifetime continues to increase with temperature until the Leidenfrost point $d$ is reached. The point $d$ indicates the interruption of contact between the drop and the heating surface due to the formation of a stable vapor film. The range $\mathrm{c}-\mathrm{d}$ is referred to as the transition regime. With $T_{w}$ beyond the point $\mathrm{d}$, the liquid is evaporated from the heating surface by a stable layer of vapor. Hence the total evaporation time reduces slowly with subsequent increases in the surface temperature as shown by the curve $\mathrm{d}-\mathrm{e}$ which is called the spheroidal (i.e.. film-boiling type) vaporization regime.

Drops of liquid on horizontal surfaces may be classified into three kinds depending on the manner in which the drops are placed on the surface: sessile, pending and impinging drops. Pending drops have less industrial applications and thus have attracted little attention. The literature pertinent to the evaporation of liquid droplets on horizontal heated surfaces can be grouped into five categories depending on the emphasis: (i) droplet shape and size, (ii) evaporation and liquid-solid interfacial heat transfer, (iii) thermal instability and natural convection, (iv) drop spreading, and (v) drop explosion. These five problems are not separable but are intimately related.

\section{(i) Droplet Shape and Size}

Perhaps the earliest work on drops resting on a horizontal surface (sessile drops) was due to Bashforth (Bashforth and Adams, 1883). He developed a method of calculating by quadratures the exact theoretical forms of drops from the differential equation of Laplace, in 1857. The calculated forms of drops of mercury agreed well with the observed forms that he had measured in 1863. Savic and Boult (1955) presented a theory of the fluid flow associated with the impact of liquid drops with a solid surface. The shape of the spreading drop was calculated as well as the pressure distribution over the impact plane. Experiments supported the main features predicted by the theory.

The behavior of sessile drops on heated surfaces in the spheriodal vaporization regime is well documented: Aylor and Bradfield (1969) defined three major regimes of size and shape based on distinct ratios of body to surface forces: (1) There is a region over which the height of a sessile drop remains nearly constant and the volume changes through contraction along the diameter. Baumeister et al. (1966) and Borishanski (1953) separately utilized the disk model over this range, in which body forces dominate and the drop assumes the flat shape of minimum potential energy. (2) For very small drops, the ratio of body to surface forces approaches zero. Therefore, the surface forces dominate and the droplet assumes a shape of minimum surface energy. i.e., minimum surface area per unit volume, a sphere. Gottfried et al. (1966), considered the small spheroidal droplets. (3) The most physically complicated situation is in the region where both surface forces and body forces have approximately equal influence. Wachters et al. (1966) conducted an extensive work in the oblate spheriod regime.

Baumeister et al. (1966) presented a correlation for the height of a sessile drop, based on a solution of the Laplace capillary equation. Subsequently. Matchers et al. (1966) numerically solved the Laplace equation for a balance of the appropriate body and surface forces to determine the static shape of a film-boiling drop. Their calculations compared very well with the photographic data of Aylor and Bradfield (1969).

(ii) Evaporation and Liquid-Solid Interfacial Heat Transfer

Most research efforts have been directed toward the determination of the evaporation rate or heat flux across the liquid-solid interface in the spheriodal state. Borishanski (1953) developed a semi-emperical correlation for the heat transfer coefficient to liquids, treating small drops as a sphere, intermediate size to be a flat disk and large masses as a bubbly disk. By simultaneously solving the momentum, energy and continuity equations for twodimensional, radial symmetry. Baumeister et al. (1966) obtained the solutions for the heat transfer coefficient. Theory based on a flat-disk geometry agreed well with experimental data of small water droplets. Patel et al. (1966) measured evaporation times, mass thickness and bubble breakthrough dynamics in film boiling of extended masses of several liquids on a plate. Gottfried et al. (1966) experimentally determined the evaporation rate of small droplets on a stainless steel plate. Calculated evaporation times found by satisfying the heat, mass and momentum balances in an analytical model for a spherical drop agree well with experimental results. Machters et al. have developed analytical models to determine the evaporation rates of both sessile (1966a) and impinging (1966 b) drops. After neglecting the effect of radiation, simple forms of the mass, momentum and energy balances are satisfied. The calculated evaporation rates compared qualitatively well with experimental results. It was reported by Machters et al. (1966c) that in the absence of wetting, a large impinging 
drop ( $2 \mathrm{~mm}$ diameter) behaves like a rubber ball because of its elasticity due to surface tension forces, and rebounds from the surface after a short contact time. This resulted in a poor heat transfer performance between the liquid and the surface. By taking heat and momentum balance into account, Satcunanathan (1968) obtained an expression for the evaporation rate based on hemispherical drop geometry. Theory compared well with some test data (Baumeister et al. 1966; Patel and Bell, 1966). Using essentially identical model and formulation, Moriyama (1974 a) obtained an expression for the evaporation rate which is practically the same as the Satcunanathan's equation. In a separate work (Moriyama, 1974b), he measured the lifetime of water drops evaporating on a hot steel surface and derived an expression for the heat transfer rate across the liquid-solid interface.

The effects of electrostatic force, relative humidity, and heating surface temperature on the evaporation rate were investigated by Aylor and Bradfield (1969).

Researchers in combustion emphasize the droplet lifetime due to their need for applications to spray combustion. Tamura and Tanasawa (1959) measured the droplet lifetime of various liquids on a plate with surface temperatures ranging from below the boiling point to over the ignition point of the liquids.

Empirical equation

$d\left(d^{2}\right) / \mathrm{d} t=$ constant $=C_{e}$

was derived for the instantaneous droplet size $d$ as a function of time. Here, $C_{e}$ is the evaporation coefficient. The droplet lifetime is equal to the square of the initial drop diameter divided by $C_{e}$. Both Kurabayashi et al. (1967) and Teshirogi (1975) repeated the experimental study using other combustible liquids. The former work emphasized the spreading area of drops on the heated surface. The theory by Yang (1978) predicted the instantaneous drop size and lifetime for all evaporation regimes including the liquid-film, nucleate-boiling, transitionboiling and spheriodal type. The calculated results agreed well with test data by Baumeister et al. (1966), Borishansky (1953), Patel and Bell (1966) and Tamura and Tanasawa (1959) except in the transition-boiling type vaporization regime. The theoretical study was extended to the evaporation of binary liquid drops by Yang (1976, 1977). Calculated droplet lifetimes compared well with experiments by Kurabayashi et al. (1967).

\section{(iii) Thermal Stability and Natural Convection}

For submerged surfaces, the entire film-boiling problem is closely tied to Rayleigh-Taylor instability of the liquidvapor interface and the dynamics of the bubbles resulting from the periodic instability of the interface. The existence of a dense phase (liquid) supported by a light phase (vapor) is inherently unstable in a gravitational field. Any small perturbation of the interface would be expected to disrupt the interface and lead to bubble formation. However, the surface tension acts to damp out short wavelength perturbation by creating an opposing force which tends to restore the smooth interface. Large wavelength disturbances (larger than the critical wavelength) are not damped out but can exist only on surfaces having a linear dimension comparable to the wavelength. Among unstable wavelengths, some grow faster than others. The wavelength that grows most rapidly is the most dangerous one. Patel and Bell (1966) applied this idea to the film boiling of extended masses. They assumed that bubble breakthrough (the center of the drop) may be interpreted in terms of Rayleigh-Taylor instability with a characteristic wavelength between the critical and the most dangerous wavelength.

Kotake (1970) studied the unstable phenomenon of droplets on a heated plate in the early spheroidal state. His theoretical model postulated that evaporation occurs when the droplet is in direct contact with the surface as a result of instability at the liquid-surface interface. Computed lifetimes obtained through the application of a perturbation technique on the equations for momentum, heat and mass balance agreed well with the experimental values. A linear small-perturbation method was applied by Han and Yang (1980) which yielded the criterion for marginal stability in a hemispherical liquid drop on a heated surface induced by surface tension mechanism. The factors leading to greater stability were disclosed.

Recently, a simple optical procedure was developed to investigate the morphology of natural convection and the dynamic behavior of minute drops evaporating on a glass plate by means of a laser shadowgraphic device (Zhang and Yang, 1983). Figure 2 depicts the device which will be described in the succeeding section. This method is the only non-intrusive means of visualizing flow patterns in a time-varying "lens" shaped system - the evaporating drop. Through the use of this method, Zhang and Yang (1982) disclosed the existence of three distinct flow structures at

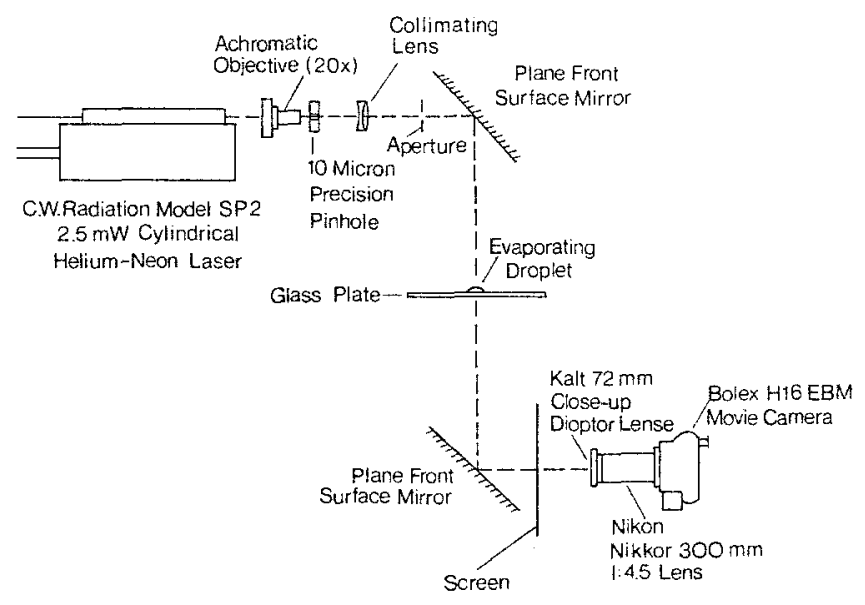

Fig. 2. A schematic of laser shadowgraphic system 
the liquid-air interface: stable-, substable- and unstabletype. The interface of stable type drops such as ethyl ether, cyclohexane and carbon tetrachloride remains smooth, with the outer rings in concentric circular form on the shadowgraph. Its drop volume-time relation can be expressed by a single curve. The surface of an unstable type drop, for example acetone, methanol or ethyl alcohol is rippled and the outer rings on the shadowgraph form irregular radiant stripes within a sawtooth-like circle. It undergoes three stages of the evaporation process: quit, vigorous and residual evaporation. The substable type drops such as ethyl acetate, benzene. chloroform and methylene chloride, have the outer rings distorted from a circular shape and spiked. Their volume-time test results fall close to a single curve. An interfacial flow map is constructed. The mechanism of ripple formation which enhances the evaporation rate is found to be caused by hydrophilicity of the liquid.

\section{(iv) Droplet Spreading}

Bascome et al. (1964) investigated the mechanisms of spontaneous spreading of liquids over unheated solids. An interference microscope was used for observation of spreading and an ellipsometer for determination of liquid film thickness. It is disclosed that the spontaneous spreading of liquids on smooth, clean metal surfaces is characterized by the advance from the bulk liquid of an invisible primary film less than $1000 \mathrm{~A}$. thick. usually followed by a visible thicker secondary film. The primary film is considered to advance largely by surface diffusion, while the movement of the secondary film results from a surface tension gradient, i.e. Marangoni effects, across the transition zone between the primary and secondary films. This gradient is produced by unequal evaporative depletion from these two regions of a volatile contaminant having a lower surface tension. If the volatile contaminant has a higher surface tension than the main component, the gradient is reversed, and the liquid recedes. Removal of the volatile constituents eliminates the secondary but not the primary spreading. Liquid may also spread by capillary flow in microscratches. The drop initially assumes a dome-shaped profile. As the liquid film advances on the surface, it develops a plateau region with a leading edge at a small angle to the solid surface. Progressively, a ridge is developed behind the edge, still maintaining an apparent contact angle with the solid. These spreading phenomena are observed on both horizontal and vertical surfaces. Molecules having highly polar functional groups are generally adsorbed on metal surfaces to form monomolecular films over which the liquid cannot spread. On the other hand, relatively low polar liquids will spread with an apparent angle of contact.

Recently, Kurabayashi and Karasawa (1976) observed the spreading behaviors of water, methanol, $\alpha$-methyl- naphthalene, and $\mathrm{n}$-heptane drops evaporating on a chrome-plated stainless steel plate with surface temperatures ranging from below the boiling point to over the Leidenfrost point. The influence of impact velocity on the drop lifetime was determined.

\section{(v) Drop Explosion}

A vapor explosion or explosive boiling may result when a very hot liquid, such as molten salts, $\mathrm{UO}_{2}$, steel or aluminum, is rapidly mixed with a colder, vaporizable liquid such as sodium or water. It also occurs when cold drops are placed on a hot solid surface. These processes are referred to as fuelcoolant interactions (FCI) which are of great concern to the safety of nuclear reactors, liquefied gas plants, molten metal processing, pulp industry and heat treatment. Vapor explosions are classified into two groups according to the speed of heat transfer between the two media: A large-scale vapor explosion involves two liquids at temperatures so different such that the heat is transferred on a time scale comparable or less than the acoustic relief time of the liquid-liquid system. A smallscale vapor explosion refers to the case in which energy is stored within the cold liquid on a time scale longer than the acoustic relief time. Two schools of thought prevail on the major cause in the triggering of small-scale explosions and in the triggering and propogation of large-scale explosions, which result from coarse premixing of the two liquids: spontaneous homogeneous nucleation (in the cold liquid) and film boiling destabilization. Henry and Fauske (1979) present a comprehensive survey of the literature pertinent to the problems of a vapor explosion resulting from the contact between cold and hot liquids. They proposed a spontaneous nucleation model to describe the inception and propagation mechanisms for vapor explosions: The necessary conditions for an explosive boiling include (i) the temperature threshold for spontaneous nucleation in the cold liquid, (ii) a sufficiently thick thermal boundary layer in the hot liquid to sustain vapor embryos of the critical size, implying the existence of stable film boiling prior to vapor explosion, (iii) the size of cold liquid droplets exceeding a critical value to be captured by the hot-liquid surface, and (iv) the interface temperature between the two liquids, $T_{i}$ exceeding the homogeneous nucleation value.

Inoue and Bankoff (1981) and Inoue et al. (1981) proposed the destabilization of film boiling (vapor film collapse) as the mechanics of triggering and propagation. Destabilization is favored by a decrease in the hot or cold liquid temperature (or both), increase in relative velocity of the two fluids, or by a sudden increase in pressure. Inoue and Bankoff (1981) studied experimentally the heat transfer during the first two ms after passage of a shock past a hot nickel tube surrounded by subcooled Freon-113 or ethanol. The study concluded that (i) the heat transfer 
in triggering and early propagation of vapor explosions occurs by rapid fragmentation and formation of new contact area during the elementary propagation cycle, (ii) the nucleation and bubble growth phenomena are irrelevant to the triggering of vapor explosions, a denial of the spontaneous nucleation mechanism, and (iii) only partial contact is made upon collapse of the vapor film which was rapid and produced by relatively weak shocks. They suggested that vapor explosions are nearly always propagated by boiling, or boiling-like, mechanisms and that the principal mechanism for rapid mixing is bubble formation and collapse. A simplified model for local mixing due to local variations in contact pressure is the "splash" theory of Ochiai and Bankoff (1976). The theory concluded that "splash" (instantaneous boiling or vapor explosion) occurs within the temperature range $T_{s n}<T_{i}<T_{c r}$. Here, $T_{s n}$ and $T_{c r}$ denote the spontaneous nucleation and critical temperatures of the cold liquid, respectively, while $T_{i}$ signifies the interfacial contact temperature. Inoue et al. (1981) investigated analytically the vapor film collapse in order to gain some insight into the mechanism of vapor explosions. It was disclosed that the initial vapor mass is a key variable determining whether collapse is achieved. The analytical results were in general agreement with the experimental data.

A recent study of Takaki and Shoji (1982) disclosed a substantial difference in behavior between the collapse of Leidenfrost boiling of single drops of ordinary liquids such as ethanol or water, on molten metal (tin) and oil (silicon) surfaces. The vapor explosion in the liquid-liquid system was characterized by a long waiting time (order of seconds depending on the hot surface temperature), while that in the molten metal-liquid system was instantaneous. These vapor explosions were found to occur only within a certain range of the hot liquid temperature depending upon the degree of subcooling, drop size and Weber number (drops were released from a height). The temperature range deviated significantly from that derived by the "splash" theory (Ochiai and Bankoff). Inoue and Bankoff (1978) reported little difference in behavior between the collapse of ordinary liquid drops on molten Woods metal or solid metal surfaces. Ogiso et al. (1982) studied the evaporation and vapor explosion of liquid drops on molten salts of binary mixtures of various compositions. It was disclosed that (i) vapor explosions occur when the molten salt temperature is between the melting and eutectic points, that is, when both the liquid and solid solutions co-exist, and (ii) the higher the probability for occurrence of a spontaneous boiling, the more violent is the vapor explosion. They suggested that both the latent heat of drops and the surface conditions of molten salts are related to the mechanism of vapor explosions. In the case of a pure saltliquid system where stable film boiling occurs, no vapor explosion is possible because the Leidenfrost point of the cold liquid is lower than the melting point of the pure salt.

lida et al. (1982) conducted experiments on vapor explosions resulting from mixing a molten salt drop $(10 \mathrm{~g})$ such as $\mathrm{LiCl}$ or $\mathrm{LiNO}_{3}$ in water (bath). It was concluded that (i) stable film boiling and $T_{i}>T_{s n}$ are necessary conditions for vapor explosions in the molten salt-liquid system and (ii) both the hot and cold liquids have the upper and lower temperature limits for vapor explosions.

All the studies mentioned above confirm that stable film boiling is a necessary condition for vapor explosions which take place only within a certain temperature range depending upon the combination of the hot (molten salts and metal or liquid) and cold liquids. Which of the two theories, namely the destabilization of film boiling and the spontaneous nucleation-growth phenomena, describe the inception and propagation mechanisms for explosions is not yet certain.

This paper deals with both the evaporation and explosion of drops in contact with a heated plate. The flow patterns in different vaporation regimes are determined by means of the laser shadowgraphic and direct photographic methods.

\section{Experiments}

Two experimental methods were employed in the study: laser shadowgraphy and direct photography. In the latter method, the flow patterns in the evaporating drops were made visible with fine aluminum powder. The behavior of these drops was investigated in all four heating regimes: liquid-film, nucleation-boiling. transition-boiling and spheriodal type vaporization.

\subsection{Laser Shadowgraphy}

The shadowgraphic arrangement consisted of an optical system, a circular thermofoil heater, a test object, and a photographic equipment, as shown in Fig. 2. The test object was a transparent medium, namely a minute drop of evaporating liquid, on an optically flat quartz glass plate, $102 \mathrm{~mm} \times 127 \mathrm{~mm}$ in size and $3 \mathrm{~mm}$ thick. The optical system consisted of a light source, an achromatic objective, a pinhole, an aperture, a pair of surface mirrors, a screen and a collimating lens.

A C. W. Radiation Model SP 2, $2.5 \mathrm{~mW}$ cylindrical helium-neon laser was used as the light source. The $20 \times$ achromatic microscopic objective was mounted on a frame together with the 10 -micron precision pinhole. The collimating lens was placed at its focal length from the pinhole. These four components were aligned on a horizontal bench. Invariably a laser beam contains diffraction noise, flashlamp, or plasma illumination and transverse mode structure. Much of this can be removed by a procedure of spatial filtering - the beam is focused by a high quality lens, the objective, through the tiny pinhole used to control apertures of laser beams and as a spatial filter. The parallel beam of light emerging from the lens 
was made to pass through the evaporating drop from top to bottom (thus producing a top view of the interfacial activity) by an arrangement of two plane front surface mirrors, $102 \mathrm{~mm} \times 127 \mathrm{~mm}$ and $6 \mathrm{~mm}$ thick as shown in Fig. 2. A circular thermofoil heater with $18 \mathrm{~mm}$ ID and $66 \mathrm{~mm}$ OD was adhered on to the under side of the glass plate. The Minco Model H-277 (B) heater with 85 ohms resistance had an output capacity of 99 watts (at $24^{\circ} \mathrm{C}$ ), capable of heating up a minute drop to explode in the transition-boiling vaporization stage. The two mirrors were aluminized on the surface nearest the objects to be viewed so that light may be reflected with no refraction. They were mounted in parallel on a specially-designed frame at 45 degrees. The test plate was inserted into the vertical light beam between the two. The mirror arrangement was used to obtain a horizontal view of the evaporating drop. The optical path from the lower mirror was horizontal into a 16-mm Bolex H16 EMB movie camera having a Nikon Nikkov $300 \mathrm{~mm} 1: 4.5$ lens. A Kalt $72 \mathrm{~mm}$ Close-Up Dioptor lens was mounted on top of the Nikon lens to photograph the air-liquid interface of an evaporating drop. A drop was carefully placed on the test plate by means of a $50-\mu 1$ Monoject micro syringe. The needle tip was touching the plate surface before and during the injection to permit the formation of a calm, unsplashing drop.

A freshly polished (wiped with dry clean cotton balls) quartz glass plate would not hold unstable-type liquid drops which would spread over it as a thin film. To maintain consistency and for reproduction of the test results, each experiment was conducted at least 24 hours after wiping both the glass and copper (in Section 2.2) plates with dry clean cotton balls. The test surface was covered by a very soft tissue paper over the waiting period during which the surface became free of residual liquid molecules while remained intact from impurity in the ambient air.

\subsection{Direct Photography}

For effectiveness in achieving a desired temperature level, two heated surfaces were prepared for the direct photography of evaporating drops: a thin glass plate and a concave-surfaced metal block. While the former was preferred for a rapid heating in the moderate-temperature vaporization regimes, the latter was needed for evaporating drops in the transition-boiling and spheroidal regimes.

\subsubsection{Thin glass plate}

Figure 3 a illustrates the apparatus for direct photography of drops evaporating on a thin glass surface. It consisted of a white light source, a glass plate coated with developed emulsion on one side, a thermofoil heater attached onto
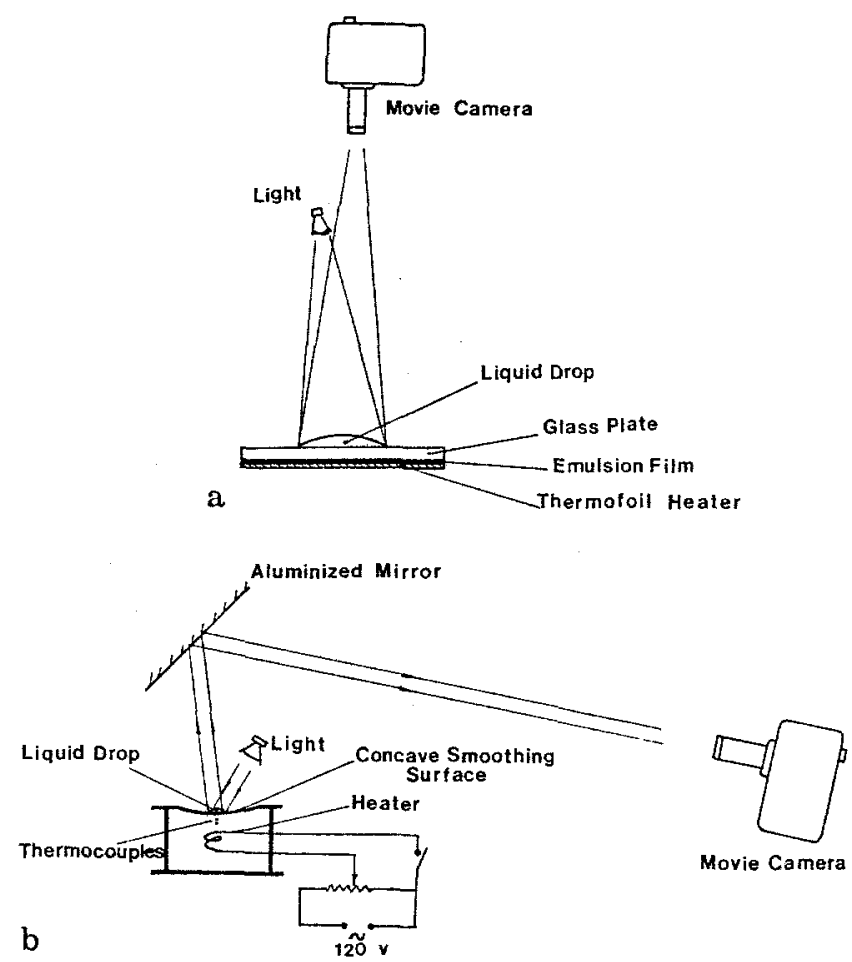

Fig. 3a and b. A schematic of direct photographic systems for drops evaporating on (a) a thin glass plate and (b) a concavesurfaced metal block

the emulsion surface, and a movie camera. The developed emulsion has a specific density such that the photographic density was approximately 1.7. The Minco HK 913 type thermofoil heater with $360 \mathrm{ohms}$ was rated at the maximum output of 39.1 watts (at $25^{\circ} \mathrm{C}$ ) and could achieve the maximum temperature of $232^{\circ} \mathrm{C}$. The merit of this test setup was to heat up the test surface to the liquid-film and nucleate-boiling vaporation regimes in a few minutes. However, the system was not suitable for higher temperature experiments due to the output capacity of the thermofoil heating tapes and the cracking of the glass plate at high temperatures. When the light source and the camera were situated at proper positions, the process of drop vaporization could be recorded clearly in photos with sharp contrast.

A tiny amount of aluminum pigment 1400 was spread on the test plate. A minute drop of $8 \mu l$ size was carefully placed on the aluminum powder using the micro syringe. The process may be reversed, that is, sprinkling the aluminum particles on a drop being placed on the glass surface. Both procedures eventually produced a fine suspension of solid particles in an evaporating drop.

\subsubsection{Concave-Surfaced Metal Block}

Figure $3 \mathrm{~b}$ shows another heating system with higher heat capacity for the vaporization experiments in the transition- 
boiling and spheroidal heating regimes. It takes a few hours of heating time to reach a higher temperature level. The system consisted of a copper block with its lower end in direct contact with a resistance heater. Both the block and the heater were placed within a metal container filled with an insulating material. The exposed end of the copper block was chrome-plated and concaved so that a drop might not drift away from the surface under the spheroidal vaporation stage. Two iron-constantane thermocouples were implanted along the center line of the circular copper block at different depths from the chromeplated test surface. Both the temperature and the heat flux at the center of the copper surface can be determined by an extrapolation of the two temperatures measured at different positions. An aluminized mirror was placed over the heated surface at a proper orientation in relation to the light source so that a drop evaporating on the surface can be photographed by the movie camera. The same procedure as mentioned in 2.2.1 was employed to suspend aluminum powder in evaporating drops.

\section{Results and Discussion}

Various liquids at low boiling point were studied: ethyl ether, cyclohexane and carbon tetrachloride known as the stable type liquids at room temperature; ethyl acetate, benzene. chloroform and methylene chloride of the substable type: and acetone, methanal and ethyl alcohol in the unstable category. Each liquid was tested in all four heating stages: liquid-film, nucleation-boiling, transitionboiling and spheroidal vaporation regimes. The flow structure in the evaporating drops was observed through both the laser shadowgraphic and direct photographic methods. Some typical results are depicted in Figs. 4, 6 through 10 . It is important to note that the interfacial flow map, a plot of the Marangoni number Ma versus the dielectric constant $\varepsilon$, defines the domain of three basic interfacial flow structures. Since both $\mathrm{Ma}$ and $\varepsilon$ tend to decrease with an increase in the liquid temperature, the flow structures in an evaporating drop may change from the unstable to substable type or from the substable to stable type as the heating of drops is increased.

\subsection{Liquid-Film Type Vaporization Regime}

This regime corresponds to the $a-b$ range in which the heating surface temperature $T_{w}$ is below the liquid saturation temperature $T_{b}$ (corresponding to point $\mathrm{b}$ in Fig. 1). The natural convection and thermal stability phenomena are reported in Zhang and Yang (1982) and will not be repeated here. When $T_{w}$ was maintained at room temperature or slightly above, the laser shadowgraph disclosed three interfacial flow structures: unstable, substable and stable. In this study, the direct photographic

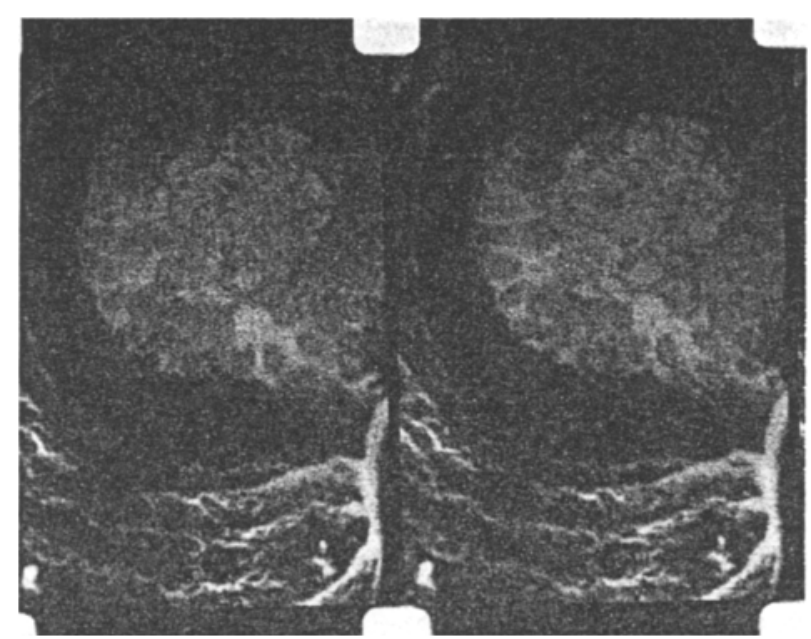

Fig. 4. Flow pattern in a chloroform drop under liquid-film type vaporization process

system revealed the existence of four flow regimes in the stable and substable type evaporation: stagnant, buoyancy driven. surface tension driven and cellular motion regimes. Figure 4 depicts the flow patterns in a chloroform drop, obtained using the system of Fig. $3 \mathrm{a}$. The white stripes at the bottom are the traces of aluminum particles left behind after the drop edge receded upward with evaporation. Different liquid exhibited different cellular patterns. In the same drop. the cell size changed with time, smaller in the initial stage of evaporation but growing slightly thereafter.

Figure 5 is a schematic diagram of the flow pattern that appeared in Fig. 4. The flow field may be divided into four regions. Region I was filled with intensive natural convection currents, while the ring-shaped region II consisted of a few layers of cellular structure. Located at the summit of the drop was the stagnation region III. A very weakly circulating region IV existed at the bottom of the drop. As evaporation proceeded, region I shrank, while the cellular structure in region II moved toward region III. Natural convection currents were so strong that no distinct flow regime was observed in the unstable type drop. However, with an increase in $T_{w}$, the buoyancy effect was enhanced to promote the strength of natural convection

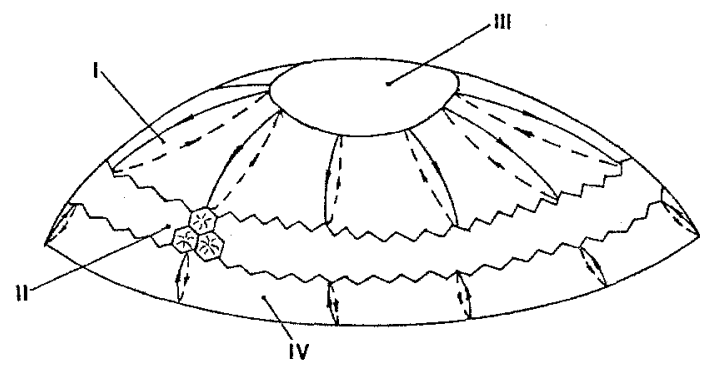

Fig. 5. A schematic diagram of flow pattern in a stable or substable drop under liquid-film type vaporation process 
currents, resulting in a reduction of the flow regimes in the stable and substable type drops from four to two, regions I and III.

\subsection{Nucleate-Boiling Type Vaporization Regime}

The nucleation and bubble growth phenomena occur inside the drop, when $T_{w}$ takes a value between $T_{b}$ and $T_{c}$, the maximum boiling rate point (point $c$ in Fig. 1). Figure 6 depicts a shadowgraphic result of a stable-type, cyclohexane drop. The round dark spot at the photo center was the shadow of the drop while the surrounding dark circle represented the outer edge of the aperture in Fig. 1. The laser beam cast a bright region around the dark circle. The outermost periphery portrayed the flow structure at the air-liquid and liquid-solid interfaces of the drop. $T_{n}=81^{\circ} \mathrm{C}$ is slightly over $T_{b}=80.9^{\circ} \mathrm{C}$. Similar situations for a substable-type, benzene $\left(T_{b}=80.3^{\circ} \mathrm{C}\right)$ with $T_{w}=80.5^{\circ} \mathrm{C}$ and an unstable-type. acetone $\left(T_{b}=56.4^{\circ} \mathrm{C}\right)$ with $T_{w}=57.1^{\circ} \mathrm{C}$ are shown in Figs. 7 and $8 \mathrm{~b}$, respectively. The outer periphery of all three types formed a slightly distorted circular shape. The sawtooth like outer rings, an important feature of an unstable-type drop under the liquid-film type vaporation process, did not appear in Fig. 8 a because the characteristics of drop evaporation has changed as a result of reduction in Ma and $\varepsilon$ with an increase in the liquid temperature. Similarly, Fig. 7 deviates from the typical shape of a substable-type drop evaporation in the liquid-film type vaporization regime. One should note cellular structures in the vicinity of the outer periphery on the shadowgraph. It is theorized that they were induced by the fluid motion resulting from nucleation or bubble growth within the drop. The observation of fluid motion by means of aluminum pigment suspension revealed vigorous fluid motion in the drop. It was not able to identify bubbles in the drop by the present methods. At higher temperatures in the nucleate-boiling type vaporization regime, difficulty arose in the use of the laser shadowgraphy for two reasons: (i) the drop geometry

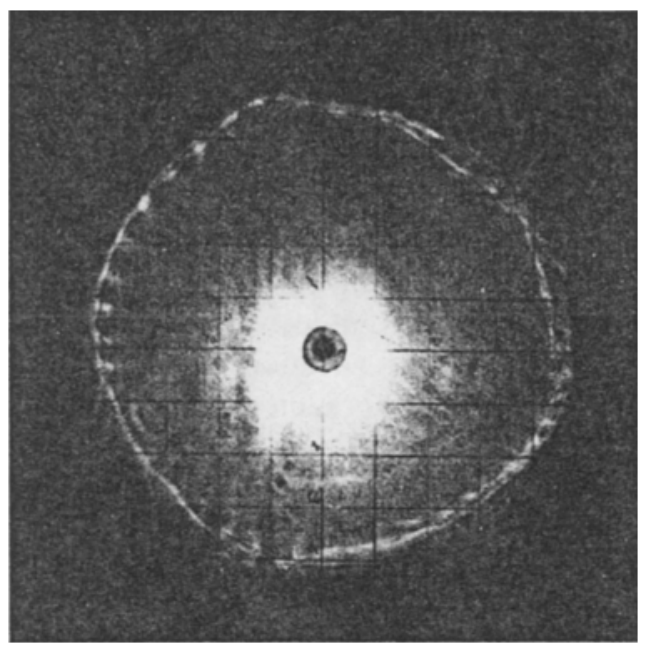

Fig. 7. Instant laser shadowgraph of a substable-type. benzene drop under nucleate-boiling type vaporization process $\left(80.5^{\circ} \mathrm{C}\right)$
Fig. 6. Instant laser shadowgraph of a stable-type, cyclohexane drop under nucleate-boiling type vaporation process $\left(81^{\circ} \mathrm{C}\right)$
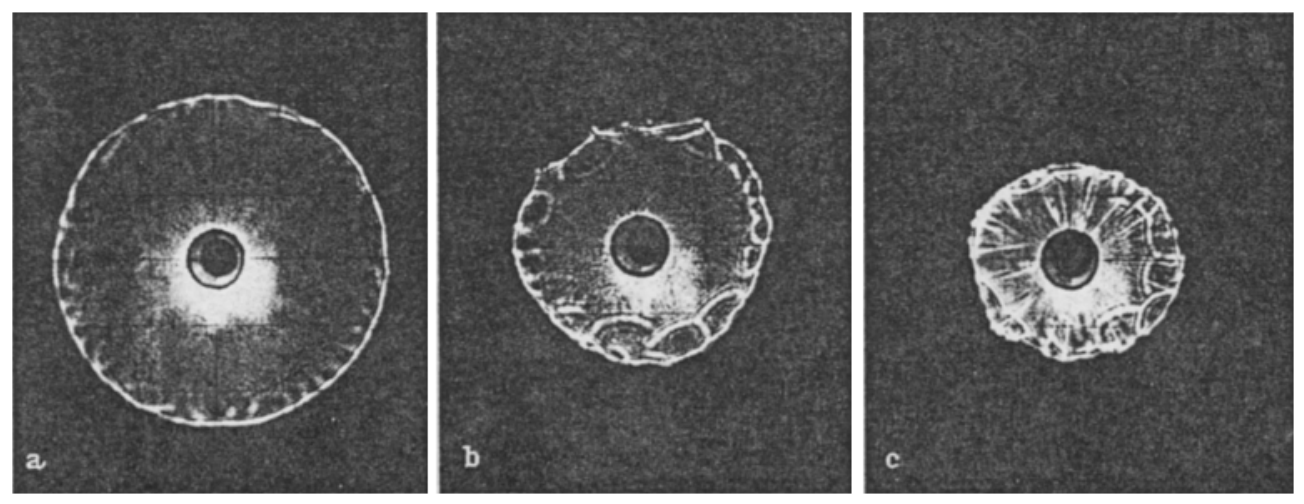

Fig. 8a-c. Instant laser shadowgraph of an unstable-type acetone drop under liquid-film type vaporization process (a) acetone $54^{\circ} \mathrm{C}$ and nucleate-boiling type vaporization process (b) $57.1^{\circ} \mathrm{C}$ and (c) $62^{\circ} \mathrm{C}$ 


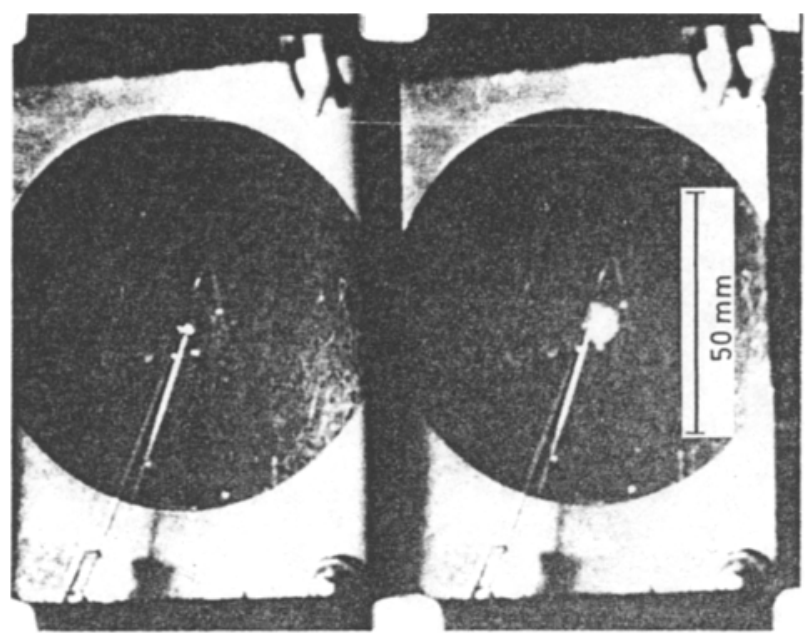

Fig. 9. Consecutive direct photographs at 25 frames/second prior to and at the explosion of a benzene drop under transition-boiling type vaporization process

deviates from the lens shape required in the application of this optical method, and (ii) the drop is in vigorous shaking and vibrating motion induced by the growth of bubbles inside the drop.

\subsection{Transition-Boiling Type Vaporization Regime}

When the surface temperature $T_{1}$ was set in the range between $T_{c}$ and $T_{d}$. the Leidenfrost point, the drop vaporization is said to be in the transition-boiling type regime. A drop exploded immediately on contact with the heating surface. Figure 9 depicts the direct photographs at 25 frames per second prior to and at the explosion of a benzene drop on the heating surface in Fig. $3 \mathrm{~b}$. The needle was faintly visible with its shadow appearing as a bright

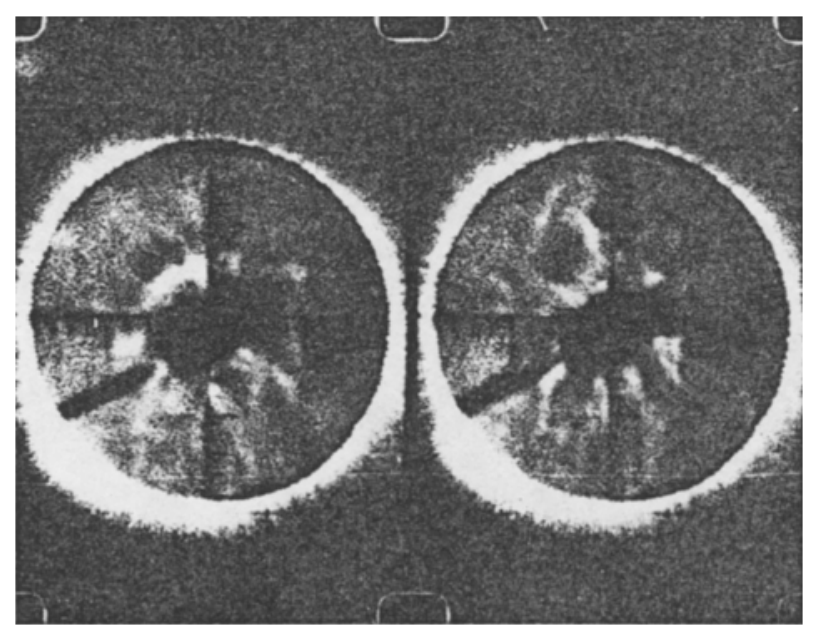

Fig. 10. Consecutive laser shadowgraphs at 25 frames/second showing the explosion of a benzene drop under the transitionboiling type vaporization regime
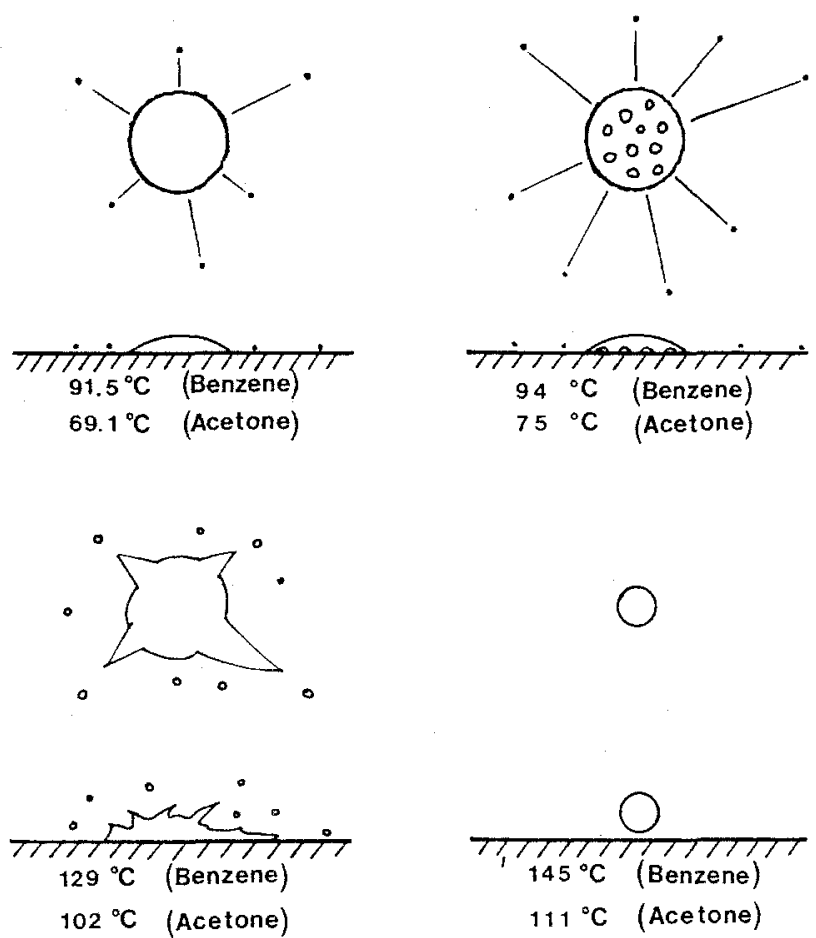

Fig. 11. A schematic of drop behavior under various heating regimes

straight line. A scale was provided for estimating the dimension. A movie of the laser shadowgraph of a benzene drop was taken at 25 frames per second. Figure 10 shows the image of the drop at two consecutive instants during the explosion. The dark spot at the center was the shadow of the exploding drop and the surrounding white stripes were the cloud of vapor resulting from the explosion. The bright ring surrounding the circular image was due to the diffraction of the laser light.

It is disclosed for the first time that drop explosion on a hot surface (solid, molten metal and salt and liquid) occurs in the transition-boiling type vaporization regime. In other words, $T_{w}$ must be within the range of $T_{c}$ and $T_{d}$. This observation is in support of the spontaneous nucleation model for the mechanism of drop explosion (Henry and Fauske, 1979).

\subsection{Film-Boiling Type Vaporization Regime}

When the surface temperature is at or above the Leidenfrost point, the drop floated on a very thin vapor film. The drop taking a spherical form never exploded but evaporated rather slowly. The laser shadowgraphy is not appropriate for this drop geometry. One important observation was that the drop exploded whenever it rolled on to a micro-scratch on the heating surface in Fig. $3 \mathrm{~b}$. This observation supports the theory that the destabilization of film boiling is the mechanics of triggering drop explosion (Inoue and Bankoff, 1981). Figure 11 is a schematic sum- 
marizing the shape and behavior of a drop in different vaporization regimes. Small circles are extremely tiny drops barely visible by the naked eye shooting out from the edge of the mother drop.

\section{Conclusions}

An optical method and a plain photography aided by aluminum powder suspension have been employed to investigate thermal stability and flow behavior in drops evaporating under various heating regimes. In the low liquid-film type vaporization stage, four flow regions including one with cellular motion are identified in the stable and substable drops. As the surface temperature $T_{k}$ is increased, the flow regions are reduced to two. A vigorous flow motion exists in the unstable drop. The interfacial flow structures change in the nucleate-boiling type vaporization regime due to a reduction in the Marangoni number and the dielectric constant of the liquid. An evidence of bubble growth is detected in the evaporating drops. For the first time. the microexplosion of drops on a heating surface is found to occur in the transition-boiling type heating regime. No drop explosion can take place in the spheroidal vaporization regime except when the spherical drop rolls on to a microseratch on the surface. The mechanics for triggering drop explosion includes the spontaneous nucleation-bubble growth phenomena and the destabilization of film boiling.

\section{References}

Aylor, D.: Bradfield, W. S. 1969: Effects of electrostatic force, relative humidity, heating surface temperatures and size and shape on droplet evaporation rate. I \& EC Fund. 8, 8-16

Bascon. W. D.; Gottington, R. L.; Singleterry. C. R. 1964: Dynamic surface phenomena in the spontaneous spreading of oils on solids. In: Contact angles, wettability and adhesion (ed. R. F. Gould), Adv. Chem. Ser. Vol. 43. pp. 355-379. Washington, D.C.: American Chemical Society

Bashforth. F.; Adams, J. C. 1883: An attempt to test the theories of capillary action. Cambridge: Cambridge University Press

Baumeister, K. J.: Hamill, T. D.; Schwartz. F. L.: Schoessow, G. J. 1966: Film boiling heat transfer to water drops on flat plate. AlChE Symp. Ser. 62, No. 64, 52-61

Borishansky. V. M. 1953: Heat transfer to a liquid freely flowing over a surface heated to a temperature above the boiling point. In: Problems of heat transfer during a change of state (ed. S. S. Kutateladze). USAEC TR-3405, pp. 109-144

Gottfried, B. S.; Lee, C. J.; Bell, K. J. 1966: The Leidenfrost phenomenon: film boiling of liquid droplets on a flat plate. Int. J. Heat Mass Transfer 9, 1167-1187

Han, J. C.; Yang, Wen-Jei 1980: Thermal instability in liquid droplets on a heated surface. J. Heat Transfer 102, 581-583

Henry, R. D.; Fauske, H. K. 1979: Nucleation processes in large scale vapor explosions. J. Heat Transfer 101, 280-287

Iida, Y.; Takashima, T.; Watanabe, T.; Ohura, H.; Ogiso, C.; Araki, N. 1982: A fundamental study of thermal interactions of molten salt and cold liquid. Proc. 19th National Heat Transfer Symp. of Japan, Nagoya, $511-513$
Inoue, A.; Bankoff, S. G. 1978: Destabilization of film boiling due to arrival of a pressure shock, Part I Experimental Report No. C00-2512-13, Chem. Eng. Dept., Nortwestern Univ., Evanston, Illinois

Inoue. A.; Bankoff, S. G. 1981: Destabilization of film boiling due to arrival of a pressure shock: Part I Experimental. J. Heat Transfer 103, 459-464

Inoue, A., Ganguli, A.; Bankoff, S. G. 1981: Destabilization of film boiling due to arrival of a pressure shock, Part II Analysis. J. Heat Transfer 103, 465-471

Koschmieder, E. L. 1967: On convection under an air surface. J. Fluid Mech. 30, 9-18

Kotake, S. 1970: Unsteady phenomena in evaporation of liquid droplets on heated surfaces. Trans. Jpn. Soc. Mech. Eng. 287, $1145-1152$

Kurabayashi, T.: Karasawa, T.: lida, K. 1967: Impact of liquid droplets on a solid surface. Jpn. Soc. Mech. Eng., Preprint No. 175, $153-156$

Kurabayashi, T.; Karasawa, T. 1976: Behavior of impacting methanol drops on a heated surface. Preprint of 15 th Japan. Automobile Research Institute Research Meeting. Ibaragi. Japan

Moriyama, A. 1974a: Evaporation rate of a single water droplet on hot solid surface. Tetsu-to-Hagane (Iron and Stecl). Trans. Inst. Steel Iron Jpn. 14. 290-295

Moriyama, A. 1974b: Heat Transfer from hot steel surface to a water droplet. Tetsu-to-Hagane (Iron Steel). Trans. Inst. Steel Iron Jpn. 14, 285-289

Ochiai, M.: Bankoff. S. G. 1976: A local propagation theory for vapor explosion. Paper SNI 6/3, Proc. Third Special Meeting on Sodium/Fuel Interactions in Fast Reactors, Tokyo

Ogiso, C.: lida. Y.: Araki. N.: Miyake. H.: Uehara, Y. 1982: A study of evaporation time of water droplet on molten salts in binary systems and vapor explosion. Proc. 19 th National Heat Transfer Symp. of Japan. Nagoay. 508-510

Patel, B. M.; Bell, K. J. 1966: The Leidenfrost phenomenon for extended liquid masses. AIChE Symp. Ser. 62. No. 64. 62-71

Satcunanathan, S. 1968: Evaporation rates of liquid droplets evaporating in the spheroidal state on a hot surface. $J$. Mech. Eng. Sci. 10, 438-441

Savic, P.; Boult. G. T. 1955: The fluid flow associated with the impact of liquid drops with solid surfaces. NRC MT-26. National Research Council, Canda

Scanlon, J. N.: Segel, L. A. 1967: Final amplitude cellular convection induced by surface tension. J. Fluid Mech. 30 , $149-162$

Takaki, N.; Shoji. M. 1982; Behaviors of hot liquid and volatile cold liquid at the impact. Proc. 19th National Heat Transfer Symp. of Japan, Nagoya, 514-516

Tamura, Z.; Tănasawa, Y. 1959: Evaporation and combustion of a drop contacting with a hot surface. 7th Symp. (Int.) on Combustion London: Butterworths Scientific Publications. 509-522

Teshirogi, N. 1975: Combustion control Diesel engines by heated surfaces. Doctor of Engineering Thesis, University of Tokyo, Japan

Wachters, L. H. J.; Bonne, H.; van Nouhuis, H. J. 1966a: The heat transfer from a hot horizontal plate to sessile water drop in the spheroidal state. Chem. Eng. Sci. 21, 923-936

Wachters, L. H. J.; Smulders, L.; Verneulen, J. R.; Kleiweg, H.C. $1966 \mathrm{~b}$ : The heat transfer from a hot wall to impinging mist droplets in the spheroidal state. Chem. Eng. Sci. 21, $1231-1238$

Wachters, L. H. J.; Westerling, N. A. J. 1966c: The heat transfer from a hot wall to impinging water drops in the spheroidal state. Chem. Eng. Sci. 21, 1047-1056 
Yang, Wen-Jei 1975: Theory on vaporization and combustion of liquid drops of pure substances and binary mixtures on heated surfaces ISAS Report No. 535 (Vol. 40, No. 15), Institute of Space and Aeronautical Science, University of Tokyo, Japan

Yang, Wen-Jei 1976: Nucleate-boiling type evaporation of binary liquid drops on heated surfaces. Lett. J. Heat. Mass. Transfer $3,467-474$

Yang, Wen-Jei 1977: Vaporization and combustion of binary liquid drops on heated surfaces. Proc. 1977 Tokyo Joint Gas Turbine Congress, 77-85

Yang. Wen-Jei 1978: Vaporization and combustion of liquid drops on heated surfaces. In: Two-phase transport and reactor safety (eds. T. N. Veziroglu and S. Kakac), pp. 51-67. Washington, D.C.: Hemisphere Publishing Corp

Zhang, N.; Yang, Wen-Jei $1983 \mathrm{a}$ : Visualization of evaporative convection in minute drops by laser shadowgraphy. Review Scientific Instruments 54, 70-73

Zhang, N.; Yang, Wen-Jei 1982: Natural convection in evaporating minute drops. J. Heat Transfer 104, 656-662

Received November 8, 1982

\title{
Technical Notes
}

\section{Direct Velocity Measurement in Low-speed Water Flows by Double-wire Hydrogen-bubble Technique}

\author{
Y. Iritani, N. Kasagi and M. Hirata \\ Department of Mechanical Engineering, University of Tokyo, Bunkyo-ku, Tokyo 113, Japan
}

\section{Introduction}

A new method for direct visual determination of velocity in low-speed water flows is proposed and tested. This ready-to-read method utilizes hydrogen-bubble tracers generated by the two electrode wires which are apart in the streamwise direction. The velocity is promptly determined by a simple principle without photography when two bubble sheets downstream of the wires are judged to be superimposed at the point of observation. The present method can be easily installed into experimental facilities and used for setting up the prescribed water velocity in the experiment. Associated uncertainty, for instance, is estimated to be less than $\pm 2 \%$ at $20: 1$ odds when the velocity is less than $200 \mathrm{~mm} / \mathrm{s}$.

\section{Principle}

As shown in Fig. 1, a couple of fine wires are positioned perpendicular to the flow direction with the streamwise distance of $L$. In addition, these wires are separated by the distance of $\delta$ in the transverse direction so that the wakes behind the wires will not interfere each other. These two wires are used as negative electrodes for generating fine hydrogen-bubbles, while a positive electrode of metal can be at any convenient place in the water channel. By using a DC-pulse generator, rectangular DC-pulse voltage of duration $T_{d}$ is imposed periodically with the time interval

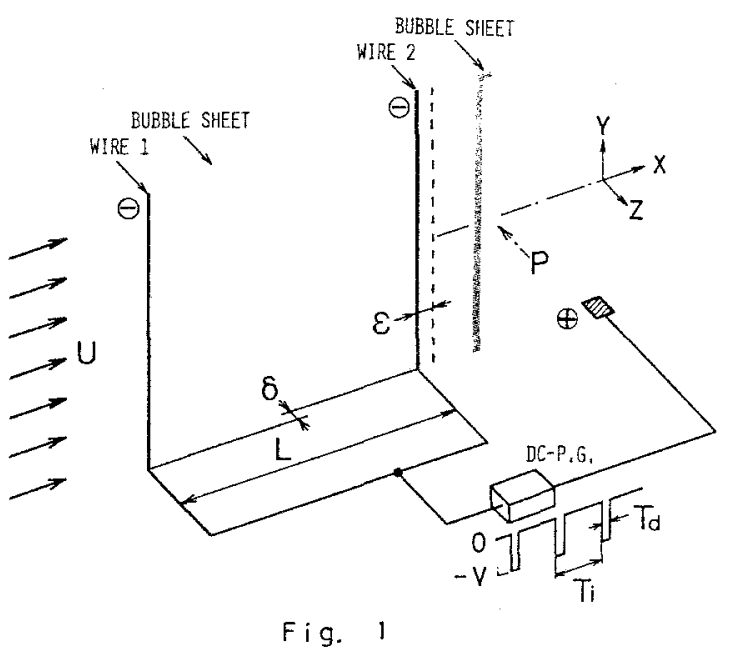

Fig. 1. Schematic of double-wire hydrogen-bubble technique

of $T_{i}$. Then, the bubble sheets are convected downstream and observed at the point of $P$ which is by more than $\varepsilon$ downstream of the downstream wire. At this point of observation the bubble speed must be virtually the same as the flow velocity. Hence, the distance $\varepsilon$ must be greater than the distance within which the bubble velocity reaches the mainstream velocity and it is estimated to be about 70 wire diameters referring to Schraub et al. (1965).

If the motion of bubble sheets downstream of the first and second wires are independent, the functional form of 